\title{
sciendo
}

\author{
BULGARIAN ACADEMY OF SCIENCES
}

CYBERNETICS AND INFORMATION TECHNOLOGIES • Volume 20, No 2

Sofia $2020 \quad$ Print ISSN: 1311-9702; Online ISSN: 1314-4081

DOI: $10.2478 /$ cait-2020-0014

\section{Analytical Overview and Applications of Modified Black- Litterman Model for Portfolio Optimization}

\author{
Todor Stoilov $v^{1,2}$, Krasimira Stoilova ${ }^{1,2}$, Miroslav Vladimirov $^{3}$ \\ ${ }^{1}$ Institute of Information and Communication Systems, Bulgarian Academy of Sciences, 1113 Sofia, \\ Bulgaria \\ ${ }^{2}$ Naval Academy, 9002 Varna, Bulgaria \\ ${ }^{3}$ Varna University of Economics, 77, Kniaz Boris bul., 9002 Varna, Bulgaria \\ E-mails: todor@hsi.iccs.bas.bg vladimirov@ue-varna.bg k.stoilova@hsi.iccs.bas.bg
}

\begin{abstract}
The paper makes analytical overviews of the Markowitz portfolio and the Capital Asset Pricing models and motivates the advances of the Black-Litterman (BL) one. This overview implies that for a small set of assets the BL model needs the characteristics of a specific market point, which cannot be taken from a global market index. The paper derives analytic relations for the new specific market point with analytical approximation of the efficient frontier. The BL model insists also expert views, which influence the portfolio solution. The paper derives formalization of the expert views from the difference between the evaluated implied returns and historical mean assets returns. Such form of expert views makes modifications of the BL model. This allows comparisons between Markowitz $(M V)$ and BL portfolio performance. Benefits of this research are demonstrated with market data and comparison of the $M V$ and BL portfolio results.
\end{abstract}

Keywords: Size Portfolio optimization, mean-variance portfolio model, BlackLitterman portfolio model, active portfolio management, decision making.

\section{Introduction}

In the portfolio practice two main strategies for the portfolio management are applied - active and passive. The passive strategy follows the market behavior. This strategy does not apply fundamental or technical analysis. As a result, the passive portfolio cannot outperform a chosen investment benchmark. The portfolio has low turnover in common, but good long-term worth [28]. The active strategy aims outperforming the market and achieving better returns by making changes of the content of the portfolio, involving quantitative analysis of the assets characteristic.

The goal of this paper is to study the peculiarities of the Black-Litterman (BL) portfolio model and to derive an algorithm for its application in active investor's strategy. The BL model is strongly influenced by the values and types of additional expert information and forecasts. Thus, different set of experts will origin different 
solutions of the portfolio model. These features of the BL portfolio model do not allow its results to be compared with the classical mean-variance portfolio problem of Markowitz. The present paper applies an approach to substitute the expert forecasts with additional data, given by the historical trends of the assets returns. Thus, applying the BL portfolio problem but using the same historical information for the mean-variance problem will have common basis for comparison of their portfolio solutions. The paper suggests new definition of the expert views from real historical data without including subjective forecasts of the experts. An algorithm for active portfolio management with the BL model is derived. The definition of the subjective view from the objective historical data of returns is presented. This modified BL portfolio model is compared with the classical mean variance one.

An active policy with low number of assets of technological companies is presented, using actual data from the stock exchange. The results achieved by the active portfolio management give preference to the modified BL portfolio model.

\section{Analytical overview of formal relations, applied in the BL portfolio}

The launch of Modern Portfolio Theory (MPT) is assumed to be one of the most important achievements in financial engineering. This theory gives solutions for diversification of investment risk and a concept for optimization of portfolio return. The goal of the portfolio optimization is to invest now and to achieve maximal portfolio return at the end of the investment horizon. Thus, the portfolio theory has intrinsic needs to forecast the assets characteristics. Due to the stochastic nature of the assets' returns, the MPT simultaneously takes into consideration both portfolio return and portfolio risk. Thus, the portfolio theory performs maximization of return by minimizing the risk. The asset returns are formalized as mean of return for a predefined historical period and the risk is quantitatively assessed by the volatility of the historical returns. That is the reason the classical portfolio optimization to use the notation Mean Variance (MV) optimization.

\subsection{Classical mean variance portfolio problem}

The Markowitz classical portfolio problem has the following analytical description [21]

$$
\max _{\mathbf{w}}\left[\begin{array}{c}
\mathbf{E}^{\mathrm{T}} \mathbf{w} \\
\mathbf{w}^{\mathrm{T}} \boldsymbol{\Sigma} \mathbf{w} \leq \sigma_{\text {max }}^{2}
\end{array}\right] \text { or } \min _{\mathbf{w}}\left[\begin{array}{c}
\mathbf{w}^{\mathrm{T}} \mathbf{\Sigma} \mathbf{w} \\
\mathbf{E}^{\mathrm{T}} \mathbf{w} \geq E_{\min }
\end{array}\right],
$$

where $\mathbf{E}^{\mathrm{T}}=\left(E_{1}, \ldots, E_{N}\right)$ are the mean returns of the assets;

$N$ - number of assets in the portfolio;

$\boldsymbol{\Sigma}$ - the covariance matrix between the asset returns, which is the quantitative assessment of the risk and the correlations between the returns of the assets;

$\mathbf{w}^{\mathrm{T}}=\left(w_{1}, \ldots, w_{N}\right), w_{i}-$ the weight or relative part of the investment, allocated to asset $i$ as a solution of the portfolio problem;

$\sigma_{\max }^{2}$ - the maximal allowed risk for the portfolio;

$E_{\min }-$ the minimal requested return by the portfolio.

The input data $\mathbf{E}^{\mathrm{T}}, \boldsymbol{\Sigma}$ for the portfolio optimization are evaluated using historical data of asset returns. Because the returns are stochastic in nature, they strongly 
influence the input data for the optimization and different factor models are used for their estimation. This classical MV problem suffers from the sensitivity of the portfolio solutions due to the accuracy of input data $\mathbf{E}^{\mathrm{T}}, \boldsymbol{\Sigma}$. The stochastic nature of the input data results in unintuitive problem solutions. As a result, many investors consider the MV problem too impractical to be used for real investments.

Problems (1) currently are integrated in a common problem

$$
\max _{\mathbf{w}}\left[\mathbf{E}^{\mathrm{T}} \mathbf{w}-\lambda \mathbf{w}^{\mathrm{T}} \mathbf{\Sigma} \mathbf{w}\right], \quad \mathbf{w}^{\mathrm{T}}|\mathbf{1}|=1, \mathbf{w}^{\mathrm{T}} \geq 0,
$$

where $\lambda$ is the risk aversion coefficient. It takes values $[0, \infty]$. For $\lambda=0$, the investor maximizes only the return, for $\lambda=\infty$ he minimizes the portfolio risk.

When problem (2) is solved for different values of $\lambda$, the solutions give points in the Risk/Return space, which define the "efficient frontier" of portfolios. The MPT requires that the user portfolio plot along this curve.

The MV optimization problem has been complicated and extended to advance its utilization in practice. Following [11, 12, 16], the extension of (2) includes inclusion of transactional costs for the portfolio, adding additional constraints to (2), incorporation of several periods for the investment policy, solving cardinality problem of investment resources.

Despite including additional analytical constraints to the portfolio problem (2), the general form of the problem is kept as a mathematical programming one.

\subsection{Formal relations from Capital Asset Pricing Model (CAPM)}

CAPM derived new formal relations in the portfolio theory. It introduces a special portfolio, named Market one. New linear relations are introduced, which formalize additional links between the portfolio parameters and the market characteristics in equilibrium [25]: Capital Market Line (CML); Security Market Line (SML); Characteristic Line (HL).

The CML is a linear relation between the mean portfolio return $E_{p}$, and portfolio risk $\sigma_{p}$ (standard deviation) for the particular market $\left(E_{M}, \sigma_{M}\right)$ and taking into account the risk free return $r_{f}$,

$$
E_{p}=r_{f}+\frac{E_{M}-r_{f}}{\sigma_{M}} \sigma_{p}
$$

The geometrical interpretation of the CML is given in Fig. 1. The CML passes through the risk free point $\left(0, r_{f}\right)$ and the market one $\left(E_{M}, \sigma_{M}\right)$. The market point $\left(E_{M}, \sigma_{M}\right)$ is the tangent one between the CML and the "efficient frontier".

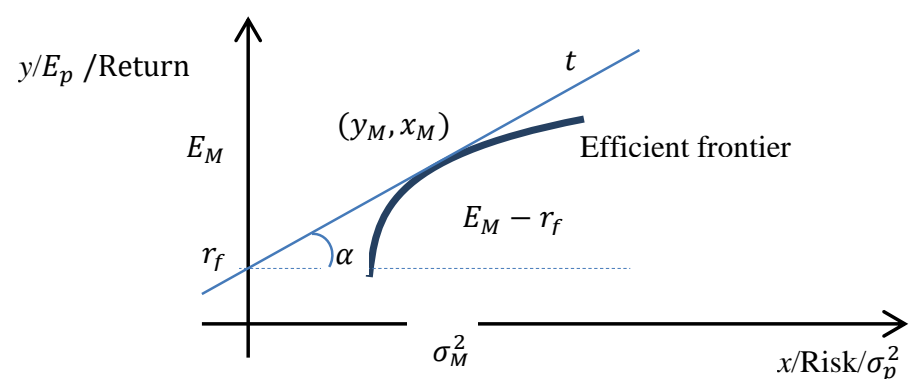

Fig. 1. Geometrical interpretation of CML 
The CML gives information about the possible portfolio characteristics (Risk and Return) in comparison to the market portfolio. In the case of having a portfolio return bigger than the market one, the investor needs to assume more risk, which is not acceptable in real investments.

The SML provides additional relation between the mean return $E_{i}$ of particular asset $i$ and the market portfolio

$$
E_{i}=r_{f}+\left(E_{M}-r_{f}\right) \beta_{i} .
$$

The coefficient $\beta_{i}$ determines the relation between the market return $E_{M}$ and the individual asset return $E_{i}$. The value of the coefficient $\beta_{i}$ is

$$
\beta_{i}=\frac{\operatorname{cov}(i, M)}{\sigma_{M}^{2}} .
$$

It is a measure of the $\operatorname{covariance} \operatorname{cov}(i, M)$ between the $i$-th asset and the market. The SML is useful to forecast the return $E_{i}$ when the market return $E_{M}$ changes to a new value. The SML allows investors to follow only the market portfolio and to assess many assets $E_{i}, i=1, \ldots, N$, is the number of assets. This relation also minimizes the computational efforts to follow $N$ assets for the portfolio management.

The cHaracteristic Line (HL) gives additional analytical relation between the current market return $R_{M}$ and the current return $R_{i}$ of asset $i$

$$
R_{i}=r_{f}+\beta_{i}\left(R_{M}-r_{f}\right) .
$$

Relation HL simplifies the forecast of the individual asset returns $R_{i}$. One can assess the market actual return $R_{M}$ and this value in a linier way defines the actual return $R_{i}$ of asset $i$. The HL uses also the well-known "coefficient beta", $\beta_{i}$, $i=1, \ldots, N$, from (5). The CAPM derives and applies in portfolio management these three relations: CML, SML, HL. These lines provide linear relations between the portfolio characteristics and the market ones. But these relations are new, additional analytical ones to the MV portfolio model. The CAPM gives useful tools to estimate portfolio characteristics, to assess possible portfolio returns, and to forecast changes in portfolio behavior.

New analytical relations have been derived by the Black-Litterman (BL) portfolio model. The idea behind this model is the estimation and forecasting the assets parameters by integrating two sources of information: the historical behavior of the assets returns and subjective forecasts of experts $[1,6,15]$. Hence, the portfolio parameters for the mean returns $E_{i}, i=1, \ldots, N$, and the covariance matrix is influenced by subjective expert views. Such integration of real history and subjective views results in portfolios, which do not contain sharp cut of types of securities, which takes place in the Markowitz portfolios. The BL model faces criticism of its efficiency [23] because the different sets of views will produce different portfolio solutions, which do not have common bases for comparisons. However, the structure of the portfolio, given by the BL model is more acceptable by the investors due to the higher level of diversification, which it gives. The analytical definition of the BL model contains several relations, which are used in the classical MV portfolio optimization. Present paper makes an overview of the BL model, which has been used for active portfolio management. 


\subsection{Definition of "implied returns" in Black-Litterman Analytical Model}

The BL model introduces new portfolio parameters, named "implied returns", $[22,24]$. They take into consideration the noises in the market, which influence the asset returns. The BL model considers the difference between the "implied returns" and the real values of the returns.

\subsubsection{Evaluation of the "implied returns"}

The "implied returns" $\Pi_{i, i}=1, \ldots, N$, are evaluated from SML analytical relation. Following (4), the SML has the following analytical description

$$
E_{i}-r_{f}=\left(E_{M}-r_{f}\right) \beta_{i} \text { where } \beta_{i}=\frac{\operatorname{cov}(i, M)}{\sigma_{M}^{2}} .
$$

The left side of (6) defines the "excess implied return" $\Pi_{i}^{*}=E_{M}-r_{f}$, thus

$$
\Pi_{i}^{*}=\frac{\operatorname{cov}(i, M)}{\sigma_{M}^{2}}\left(E_{M}-r_{f}\right) \text {. }
$$

The actual market return $R_{M}$ is a linear combination between the market capitalization weights $w_{i}^{*}$ of all assets, $i=1, \ldots, N$, and their actual returns $R_{i}$,

$$
R_{M}=\sum_{i=1}^{N} R_{i} w_{i}^{*}
$$

The same formal relation takes place for the mean values of the asset returns

$$
E_{M}=\sum_{i=1}^{N} E_{i} w_{i}^{*} \text {. }
$$

Substituting (8) and (9) in (6) for the coefficient $\beta_{i}$ it follows:

$$
\operatorname{cov}\left(R_{i}, R_{M}\right)=\frac{1}{n-1} \sum_{k=1}^{n}\left(R_{i}^{(k)}-E_{i}\right) \sum_{j=1}^{N}\left(R_{j}^{(k)}-E_{j}\right) w_{j}^{*},
$$

where $n$ are the number of data in the historical period. Changing the order of summation, it follows

$$
\operatorname{cov}\left(R_{i}, R_{M}\right)=\sum_{j=1}^{N} \frac{1}{n-1} \sum_{k=1}^{N}\left(R_{i}^{(k)}-E_{i)}\left(R_{j}^{(k)}-E_{j}\right) w_{j}^{*} .\right.
$$

The internal sum gives the co-variation between assets $i$ and $j$ or

Substituting (10) in (7), it follows

$$
\operatorname{cov}\left(R_{i}, R_{M}\right)=\sum_{j=1}^{N} \operatorname{cov}\left(R_{i}, R_{j}\right) w_{j}^{*}
$$

(11) $\Pi_{i}^{*}=\frac{E_{M}-r_{f}}{\sigma_{M}^{2}} \sum_{\mathrm{j}=1}^{N} \operatorname{cov}\left(R_{i}, R_{j}\right) w_{j}^{*}$ or in matrix form $\Pi^{*}=\lambda \operatorname{cov}(.) w^{*}$,

where $\lambda=\frac{E_{M}-r_{f}}{\sigma_{M}^{2}}$ is a parameter named "risk aversion" or market price of risk;

$$
\operatorname{cov}(.)=\left|\begin{array}{c}
\operatorname{cov}\left(R_{1}, R_{1}\right) \cdots \operatorname{cov}\left(R_{1}, R_{N}\right) \\
\ddots \\
\operatorname{cov}\left(R_{N}, R_{1}\right) \cdots \operatorname{cov}\left(R_{N}, R_{N}\right)
\end{array}\right| \text { is } N \times N \text { matrix. }
$$

The BL model applies the notation $\Sigma$ for the covariance matrix, $\Sigma=\operatorname{COV}($.). Finally, the "implied returns" $\Pi$ are

$$
\Pi=\lambda_{0} \boldsymbol{\Pi}^{*}+r_{f} .
$$

Relations (11) and (12) do not require explicit constraints for the market capitalization weights $\mathbf{w}^{*}$. But at the market point the weights always satisfy

$$
\sum_{i=1}^{N} w_{i}^{*}=1 \text {, }
$$

where $w_{i}^{*}$ gives the relative part of the investment, allocated to asset $i$. 


\subsubsection{Alternative way of definition of implied returns}

Another way for deriving relation (11), which is intensively presented in references $[16,17,27]$, is by implementing the "inverse optimization" procedure. The BL model for this case starts from the assumption that the portfolio has to maximize the return and minimize the risk. These requirements are valid also for the market portfolio and using the goal function from (2) it follows

$$
\max _{\mathbf{w}_{M}}\left\{\mathbf{w}_{M}^{\mathrm{T}} \boldsymbol{\Pi}^{*}-0.5 \lambda \mathbf{w}_{M}^{\mathrm{T}} \boldsymbol{\Sigma} \mathbf{w}_{M}\right\} \text {. }
$$

This problem lacks additional constraints, because at the market point, relation (13) is assumed to hold in implicit way. Problem (14) is an unconstrained optimization problem and its solution can be found from the first derivative

$$
\frac{d}{d w}\left(\mathbf{w}_{M}^{\mathrm{T}} \boldsymbol{\Pi}^{*}-0.5 \lambda \mathbf{w}_{M}^{\mathrm{T}} \boldsymbol{\Sigma} \mathbf{w}_{M}\right)=\mathbf{0} \text { or } \boldsymbol{\Pi}^{*}-\lambda \boldsymbol{\Sigma} \mathbf{w}_{M}=\mathbf{0},
$$

where all parameters must be known: $\lambda$ is the risk aversion coefficient; $\boldsymbol{\Sigma}$ is the $N \times N$ covariance matrix, evaluated for the historical period with data $R_{i}, E_{i}, i=1, \ldots, N ; \mathbf{w}_{M}$ is the market capitalization weights.

The parameter $\lambda$ has to be estimated according to the available data for $\boldsymbol{\Sigma}$, $\mathbf{w}_{M}$ and market characteristics $E_{M}, \sigma_{M}^{2}$.

\subsubsection{Assessment of $\lambda$}

Using (15) both sides are multiplied from the left by the vector $\mathbf{w}_{M}^{\mathrm{T}}$

$$
\mathbf{w}_{M}^{\mathrm{T}} \boldsymbol{\Pi}=\lambda \mathbf{w}_{M}^{\mathrm{T}} \boldsymbol{\Sigma} \mathbf{w}_{M} \text {. }
$$

The left side of (16) gives the market excess return $E_{M}-r_{f}$. The right side defines the market portfolio risk, $\mathbf{w}_{M}^{\mathrm{T}} \boldsymbol{\Sigma} \mathbf{w}_{M}$ or

$$
E_{M}-r_{f}=\lambda \sigma_{M}^{2}
$$

From (17) and the market portfolio characteristics it follows

$$
\lambda=\frac{E_{M}-r_{f}}{\sigma_{M}^{2}},
$$

which gives numerical value to the risk aversion coefficient for the market portfolio. Substituting (18) in (15) the "implied excess returns" are

$$
\boldsymbol{\Pi}^{*}=\lambda\left(E_{M}, \sigma_{M}^{2}, r_{f}\right) \boldsymbol{\Sigma}\left(R_{i}, i=1, \ldots, N, t \in(0, T)\right)=\frac{E_{M}-r_{f}}{\sigma_{M}^{2}} \mathbf{\Sigma} \mathbf{w}_{M},
$$

where $t \in(0, T)$ is the historical period, over which the average returns $E_{i}$ and the covariance matrix $\boldsymbol{\Sigma}$ are calculated. Respectively, the vector of "excess returns" is $\boldsymbol{\Pi}=r_{f}+\Pi^{*}$, which has to be used in the BL model.

\subsection{Formal presentation of BL model relations}

The BL model states that the vector of mean returns of the securities $\mathbf{E}_{\mathrm{BL}}$ is influenced from three components: the "implied returns" $\boldsymbol{\Pi}$; the market noise $\boldsymbol{\varepsilon}$; the subjective views of experts about the values of future asset returns.

The analytical relation, which considers the "implied returns" and the market noise, is in linear form

$$
\boldsymbol{\Pi}=\mathbf{E}_{\mathrm{BL}}+\boldsymbol{\varepsilon}, \mathbf{E}_{\mathrm{BL}}=\left|\begin{array}{c}
E_{\mathrm{BL}, 1} \\
\vdots \\
E_{\mathrm{BL}, N}
\end{array}\right| .
$$


The vector $\mathbf{E}_{\mathrm{BL}}$ is unknown and it has to be defined by minimizing the influence of the noise $\varepsilon$ and to be near to the value of the vector of "implied returns" $\Pi$. If noise is missing, $\mathbf{E}_{\mathrm{BL}}$ will be equal to the "implied returns" $\boldsymbol{\Pi}$. The components of noise $\boldsymbol{\varepsilon}=\left|\begin{array}{c}\varepsilon_{1} \\ \vdots \\ \varepsilon_{N}\end{array}\right|$ are assumed to be independent, with normal distribution and zero mean, and volatility, proportional to the volatilities of the portfolio assets

$$
\boldsymbol{\varepsilon} \sim \mathbf{N}(0, \tau \boldsymbol{\Sigma}) \text {. }
$$

These assumptions allow only the diagonal components of the co-variation matrix to be used. The value of $\tau<1$ is a scaling parameter and it defines that the volatility of the noise $\boldsymbol{\varepsilon}$ has a proportional value to the assets' volatility.

The formalization of the subjective views is given as

where $\mathbf{P}$ is a matrix, that identifies the assets, involved in the different views, $\mathbf{Q}$ is a vector with the quantitative assessments of the subject views about the changes of the asset returns, $\boldsymbol{\eta}$ is a vector noise with normal distribution, which represent the risk of the subjective views, $\boldsymbol{\eta} \sim \mathbf{N}(0, \boldsymbol{\Omega})$. The volatility of $\boldsymbol{\eta}$ is given by the matrix $\boldsymbol{\Omega}$. It is assumed that the subjective views are $k$ numbers and they are uncorrelated. Hence, the matrix $\boldsymbol{\Omega}$ is a diagonal one with $k \times k$ size. Each diagonal component $\boldsymbol{\Omega}_{i, i}$ numerically represents the volatility of the subjective view $i$. Taking into consideration (21) and (22), the unknown mean values $\mathbf{E}_{\mathrm{BL}}$ have to provide close solution to the stochastic system

$$
\begin{aligned}
& \boldsymbol{\Pi}=\mathbf{I} \mathbf{E}_{\mathrm{BL}}+\boldsymbol{\varepsilon}, \\
& \mathbf{Q}=\mathbf{P E}_{\mathrm{BL}}+\boldsymbol{\eta} .
\end{aligned}
$$

To simplify the next relations, aggregate notations are used

$$
\mathbf{Y}=\mathbf{X E _ { \mathrm { BL } }}+\boldsymbol{\psi},
$$

where

$$
\mathbf{Y}=\left|\begin{array}{l}
\Pi \\
\mathbf{Q}
\end{array}\right|, \mathbf{X}=\left|\begin{array}{l}
\mathbf{I} \\
\mathbf{P}
\end{array}\right|, \boldsymbol{\Psi}=\left|\begin{array}{l}
\boldsymbol{\varepsilon} \\
\boldsymbol{\eta}
\end{array}\right|, \overline{\boldsymbol{\Psi}}=\left|\begin{array}{cc}
\tau \boldsymbol{\Sigma} & 0 \\
0 & \mathbf{\Omega}
\end{array}\right| .
$$

Having a set of values about $\mathbf{X}$ and $\mathbf{Y}$, it is needed to find the linear regression for $\mathbf{E}_{\mathrm{BL}}$, which gives maximal approximates for (23). To find $\mathbf{E}_{\mathrm{BL}}$ according to the general least square method the Mahalonobis distance is minimized

$$
\mathbf{E}_{\mathrm{BL}}^{\min } \equiv \arg \left\{\operatorname { m i n } _ { \mathrm { E } _ { \mathrm { BL } } } \left[\left(\mathbf{Y}-\mathbf{X E}_{\mathrm{BL}}\right]^{\mathrm{T}} \overline{\boldsymbol{\Psi}^{-\mathbf{1}}}\left[\left(\mathbf{Y}-\mathbf{X E}_{\mathrm{BL}}\right]\right\} .\right.\right.
$$

The unconstrained minimization of (24) gives an analytical solution

$$
\mathbf{E}_{\mathrm{BL}}^{\min }=\left(\mathbf{X}^{\mathrm{T}} \boldsymbol{\Psi}^{-1} \mathbf{X}\right)^{-1} \mathbf{X}^{\mathrm{T}} \overline{\boldsymbol{\Psi}^{-1}} \mathbf{Y},
$$

and substituting the notations for $\mathrm{X}, \mathrm{Y}, \bar{\psi}$ the main relations in BL model follow

$$
\mathbf{E}_{\mathrm{BL}}=\left[(\tau \boldsymbol{\Sigma})^{-1}+\mathbf{P}^{\mathrm{T}} \boldsymbol{\Omega}^{-1} \mathbf{P}\right]^{-\mathbf{1}}\left[(\tau \boldsymbol{\Sigma})^{-1} \boldsymbol{\Pi}+\mathbf{P}^{\mathrm{T}} \boldsymbol{\Omega}^{-1} \mathbf{Q}\right],
$$

and volatility $\operatorname{Vol}\left(\mathbf{E}_{\mathrm{BL}}\right)=\boldsymbol{\Delta}_{\mathrm{BL}}=\left[(\tau \boldsymbol{\Sigma})^{-1}+\mathbf{P}^{\mathrm{T}} \boldsymbol{\Omega}^{-1} \mathbf{P}\right]^{-1}$.

The final $\mathrm{BL}$ vector of mean returns and the final covariance matrix are

$$
\mathbf{E}_{\mathrm{BL}}^{\mathrm{final}}=\mathbf{E}_{\mathrm{BL}}+\mathbf{r}_{\mathrm{f}} \text { and } \boldsymbol{\Sigma}_{\mathrm{BL}}^{\mathrm{final}}=\boldsymbol{\Sigma}+\boldsymbol{\Delta}_{\mathrm{BL}} \text {. }
$$

Relations (27) give new solutions $\mathbf{w}_{\mathrm{BL}}$ of the portfolio problem in comparison with the MV one, because (2) is solved with new data $\mathbf{E}_{\mathrm{BL}}^{\mathrm{final}}$ and $\boldsymbol{\Sigma}_{\mathrm{BL}}^{\mathrm{final}}$ instead of $\mathbf{E}$ and $\Sigma$ : 


$$
\max _{\mathbf{w}}\left[\mathbf{E}_{\mathrm{BL}}^{\text {final } \mathrm{T}} \mathbf{w}-\lambda \mathbf{w}^{\mathrm{T}} \boldsymbol{\Sigma}_{\mathrm{BL}}^{\text {final }} \mathbf{w}\right], \mathbf{w}^{\mathrm{T}}|1|=1, \mathbf{w}^{\mathrm{T}} \geq 0 .
$$

The value $\lambda$ of the risk aversion coefficient is different for each investor. In the case of multiple solutions of (28) by changing $\lambda \in(0, \infty)$, points of the new "efficient frontier" are found. The solution $\mathbf{w}^{\text {opt }}$ will give new structure of the portfolio in comparison to the MV problem (2). The solution $\mathbf{w}^{\mathrm{opt}}$ does not have sharp cut of values, which takes place in Markowitz portfolio.

2.5. Alternative descriptions of the formal relations in BL model

The main BL relations (26) can be presented also in less complicated forms.

2.5.1. Alternative relations for $\mathbf{E}_{\mathrm{BL}}$

Starting with (26) a zero sum of the form $\left|\begin{array}{c}0 \\ \vdots \\ 0\end{array}\right|=\mathbf{P}^{\mathrm{T}} \boldsymbol{\Omega}^{-1} \mathbf{P} \boldsymbol{\Pi}-\mathbf{P}^{\mathrm{T}} \boldsymbol{\Omega}^{-1} \mathbf{P} \boldsymbol{\Pi}$ is added, where $\mathbf{P}$ is $k \times N$ matrix, $\mathbf{\Omega}^{\mathbf{- 1}}$ is $k \times k$ matrix, $\boldsymbol{\Pi}$ is $N \times 1$ vector. The analytical sequences of transformation follow:

$$
\begin{gathered}
\mathbf{E}_{\mathrm{BL}}=\left[(\tau \boldsymbol{\Sigma})^{-1}+\mathbf{P}^{\mathrm{T}} \boldsymbol{\Omega}^{-1} \mathbf{P}\right]^{-1}\left[(\tau \boldsymbol{\Sigma})^{-1} \boldsymbol{\Pi}\right. \\
\left.+\left(\mathbf{P}^{\mathrm{T}} \boldsymbol{\Omega}^{-1} \mathbf{P} \boldsymbol{\Pi}-\mathbf{P}^{\mathrm{T}} \boldsymbol{\Omega}^{-1} \mathbf{P} \boldsymbol{\Pi}\right)+\mathbf{P}^{\mathrm{T}} \boldsymbol{\Omega}^{-1} \mathbf{Q}\right] .
\end{gathered}
$$

Using the matrix equation $\mathbf{A}^{-1} \mathbf{B}^{-1}=(\mathbf{B} \mathbf{A})^{-1}$, this gives

Relation (29) is derived in $[15,20]$. The value of vector $\mathbf{E}_{\mathrm{BL}}$ is named posterior estimate of unknown mean returns, $\boldsymbol{\Pi}$ is prior estimate (implied returns). The posterior returns $\mathbf{E}_{\mathrm{BL}}$ will be equal to the prior returns $\boldsymbol{\Pi}$ if expert views are lacking (zeros matrices $\mathbf{P}, \mathbf{Q}$ ).

2.5.2. Alternative description of the BL increase of the covariance $\Delta_{\mathrm{BL}}$

The change of the covariance matrix $\boldsymbol{\Sigma}$ in BL model is done with the additional matrix $\Delta_{\mathrm{BL}}$, analytically defined with (26). This relation can be elaborated, applying the Woodburg's matrix identity formulae

(30) $\quad(\mathbf{A}+\mathbf{U C V})^{-1}=\mathbf{A}^{-1}-\mathbf{A}^{-1} \mathbf{U}\left(\mathbf{C}^{-1} \mathbf{V A}^{-1} \mathbf{U}^{-1}\right) \mathbf{V A}$. becomes

Substituting the notations $\mathbf{A}=(\tau \boldsymbol{\Sigma})^{-1}, \mathbf{U}=\mathbf{P T}, \mathbf{C}=\mathbf{\Omega}^{-1}, \mathbf{V}=\mathbf{P}$ the value of $\boldsymbol{\Delta}_{\mathrm{BL}}$

$$
\boldsymbol{\Delta}_{\mathrm{BL}}=\boldsymbol{\tau} \boldsymbol{\Sigma}-\left[\mathbf{I}-\mathbf{P}^{\mathrm{T}}\left(\boldsymbol{\Omega}+\mathbf{P} \boldsymbol{\tau} \boldsymbol{\Sigma} \mathbf{P}^{\mathrm{T}}\right)^{-1} \mathbf{P} \boldsymbol{\tau} \boldsymbol{\Sigma}\right] .
$$

Relation (31) analytically describes that the changes in the correlation matrix $\boldsymbol{\Sigma}$ results from the components of the expert views $\mathbf{P}$ and $\boldsymbol{\Omega}$. The covariance increase $\boldsymbol{\Delta}_{\mathrm{BL}}$ is mainly influenced by the volatility of the expert views $\boldsymbol{\Omega}$, not by the forecasts $\mathbf{Q}$ for the asset returns.

\subsubsection{Analysis of the influence of matrix $\boldsymbol{\Omega}$}

Matrix $\boldsymbol{\Omega}$ influences the covariance matrix $\boldsymbol{\Sigma}$ by the component $\boldsymbol{\Delta}_{\mathrm{BL}}(\boldsymbol{\Omega})$

The BL model assumes that the volatility of the views $\boldsymbol{\Omega}$ is proportional to $\tau \boldsymbol{\Sigma}$ or

$$
\boldsymbol{\Omega}=\mathbf{P}(\tau \boldsymbol{\Sigma}) \mathbf{P}^{\mathrm{T}} .
$$


If (32) is substituted in (31), simple form of the covariance increase $\boldsymbol{\Delta}_{\mathrm{BL}}$ is derived

$$
\boldsymbol{\Delta}_{\mathrm{BL}}=\tau \boldsymbol{\Sigma}-\tau \boldsymbol{\Sigma} \mathbf{P}^{\mathrm{T}} 0.5\left(\mathbf{P} \tau \boldsymbol{\Sigma} \mathbf{P}^{\mathrm{T}}\right)^{-1} \mathbf{P} \tau \boldsymbol{\Sigma} .
$$

Applying the matrix relation $(\mathbf{A B})^{-1}=\mathbf{B}^{-1} \mathbf{A}^{-1}$, it follows:

Hence, relation (27) for the actual BL covariance matrix changes to the simple form

$$
\Sigma_{\mathrm{BL}}^{\mathrm{final}}=\boldsymbol{\Sigma}+0.5 \tau \boldsymbol{\Sigma} .
$$

2.5.4. Influence of matrix $\boldsymbol{\Omega}$ to the vector of BL mean returns $\mathbf{E}_{\mathrm{BL}}$

Using the definition of matrix $\boldsymbol{\Omega}$ from (32), the relation (29) about $\mathbf{E}_{\mathrm{BL}}$ becomes

$$
\mathbf{E}_{\mathrm{BL}}=\boldsymbol{\Pi}+0.5 \tau \boldsymbol{\Sigma} \mathbf{P}\left(\mathbf{P} \tau \boldsymbol{\Sigma} \mathbf{P}^{\mathrm{T}}\right)^{-1}(\mathbf{Q}-\mathbf{P} \Pi) \text {. }
$$

Applying the matrix equation $(\mathbf{A ~ B ~})^{-1}=\mathbf{B}^{-1} \mathbf{A}^{-1}$, it follows

Relations (33) and (34) present in simple forms the BL parameters $\boldsymbol{\Delta}_{\mathrm{BL}}$ and $\mathbf{E}_{\mathrm{BL}}$ according to the usage of relation (32).

Having new values for the asset returns $\mathbf{E}_{\mathrm{BL}}^{\text {final }}$ and covariance matrix $\boldsymbol{\Sigma}_{\mathrm{BL}}^{\mathrm{final}}$, the BL model defines another goal of the portfolio problem

$$
\max _{\mathbf{w}_{\mathrm{BL}}}\left[\mathbf{E}_{\mathrm{BL}}^{\mathrm{final} \mathrm{T}} \mathbf{w}_{\mathrm{BL}}-\lambda \mathbf{w}_{\mathrm{BL}}^{\mathrm{T}} \boldsymbol{\Sigma}_{\mathrm{BL}}^{\mathrm{final}} \mathbf{w}_{\mathrm{BL}}\right] .
$$

The unconstrained solution of this optimization gives analytical solution for the asset weights as

(35) $\frac{d}{d \mathbf{w}_{\mathrm{BL}}}(\ldots)=0=\mathbf{E}_{\mathrm{BL}}^{\mathrm{final}}-\lambda \boldsymbol{\Sigma}_{\mathrm{BL}}^{\mathrm{final}} \mathbf{w}_{\mathrm{BL}}$ or $\mathbf{w}_{\mathrm{BL}}=\left(\lambda \boldsymbol{\Sigma}_{\mathrm{BL}}^{\mathrm{final}}\right)^{-1} \mathbf{E}_{\mathrm{BL}}^{\mathrm{final}}$, where $\lambda$ is the risk aversion coefficient of the investor. Solutions (35) can be practically used if short sales are eligible and components of $\mathbf{w}_{\mathrm{BL}}$ may have negative values. In practice the portfolio problem has constraints as problem (26).

2.6. Classical definition of the expert views in the BL model (P(1) model)

The BL relations (26) contain four additional parameters, formalizing the form of the expert views:

$\mathbf{P}$ is a matrix, identifying the assets, included in the views;

$\mathbf{Q}$ is a vector, numerically giving the expert value for the future returns of assets, defined in $\mathbf{P}$;

$\boldsymbol{\Omega}$ is a diagonal matrix, which formalizes the uncertainty of the views. The expert views are assumed to be uncorrelated, which makes $\boldsymbol{\Omega}$ a diagonal one;

$\tau$ is a weighted coefficient, $\tau<1$, which is used to keep same correlation relationships between the asset returns, but the intensities of these correlations are lower in comparison to the initial covariance matrix $\boldsymbol{\Sigma}$.

The views are defined in absolute and/or relative ways. For easier understanding of this formalization, an example is given for the case of $N=4$ assets in the portfolio.

1. Absolute way of view formalization. The notations $\mathbf{P}=\left|\begin{array}{llll}1 & 0 & 0 & 0 \\ 0 & 0 & 1 & 0\end{array}\right|$, $\mathbf{Q}=\left|\begin{array}{c}2 \% \\ -4 \%\end{array}\right|$ mean that the first view states that asset one will increase its mean return with $2 \%$ and the asset four will decrease with $4 \%$. 
2. Relative way of view formalization. The notations $\mathbf{P}=\left|\begin{array}{llll}0 & 1 & 0 & -1\end{array}\right|$, $\mathbf{Q}=|3 \%|$ say that the return of asset two will outperform the return of asset four with $3 \%$. It is important to have zero sum for these values on the row of matrix $\mathbf{P}$.

The uncertainty of matrix $\boldsymbol{\Omega}$ contains the variance of the expert views. It is assumed that the views are uncorrelated and this results in a diagonal matrix $\boldsymbol{\Omega}$ $\boldsymbol{\Omega}=\left|\begin{array}{cccc}\omega_{1} & 0 & \cdots & 0 \\ 0 & \omega_{2} & \cdots & 0 \\ \cdots & \cdots & \cdots & \cdots \\ 0 & 0 & \cdots & \omega_{k}\end{array}\right|$ is the $k \times k$ matrix, $k$ is the number of expert views.

Because it is not easy to quantify the uncertainty $\omega_{i}, i=1, \ldots, k$, in $[16,22]$ is assumed that the elements of $\boldsymbol{\Omega}$ are proportional to the variance of asset returns. Therefore, the most used formalization of $\boldsymbol{\Omega}$ is

$$
\mathbf{\Omega}=\tau \operatorname{diag}\left(\mathbf{P} \boldsymbol{\Sigma} \mathbf{P}^{\mathrm{T}}\right) .
$$

The parameter $\tau$ is named "weight on views". The value of $\tau$ is accepted to be positive, less than 1. In [5] $\tau$ is chosen close to zero. In [20] $\tau$ is suggested to be related to the length of the historical period $n$, for which the asset returns are taken as initial data, $\tau=1 / n$. The period $n$ corresponds to the value of horizon, during which the mean returns are evaluated, $E_{i}=\frac{1}{n-1} \sum_{j=1}^{n} R_{i}^{(j)}, i=1, \ldots, N$.

The notation used for this model of expert views is $\mathbf{P}(1)$, because matrix $\mathbf{P}$ contains integer algebraic values of 0 and 1 .

The inclusion of expert views in BL model leads to changes of the main characteristics of the assets: their mean returns and their volatilities and covariance relations. Hence, different values of expert views as different combinations of experts will result in different portfolio problems with different portfolio solutions.

Thus, it is not possible to assess and to compare on common basis the performance of the different portfolio solutions. In the next part of this research a formal derivation of expert views is done, which are based on additional usage of information, given by the historical trends of the assets returns. Such approach allows the Markowitz and BL portfolio solutions to be compared on common basis, because they are using the same set of historical information about the assets returns. This approach is also applicable for cases, when subjective expert views are missing or not reliable.

\section{Definition of expert views in BL model from historical trend of assets returns}

Without claiming of exhaustive overview, the expert views for future levels of assets' returns are under consideration in [3, 4, 6-10, 13, 14, 19, 20].

This research applies special form for the definition of matrix $\mathbf{P}$ that modifies the BL model. The new components of $\mathbf{P}$ can take arbitrary values from $[-1,+1]$, which is a difference with the classical BL model. The notation $\mathrm{P}(\alpha)$ is used, for the non-integer, weighted values for the components of the expert views in $\mathbf{P}$.

The weighted form of views originally was introduced in [26]. It was given new interpretation of the content of matrix $\mathbf{P}$ as a risk value of special portfolio with equal weights of the assets. The resulting new values of expert views are calculated from 
additional estimation of the historical data of the assets' returns. Using the conclusion from [2] that the investor with forecasting abilities can improve the expected utility of the portfolio, here the new form of forecasting is based on additional evaluations of the differences between the implied and historical mean assets' returns. The weighted form of matrix $\mathrm{P}$ is derived, according to this difference.

Let assume that $s$-th row $\mathbf{p}_{s}$ of matrix $\mathbf{P}$ contains two components, +1 and -1 , situated respectively on $i$-th and $j$-th columns, where

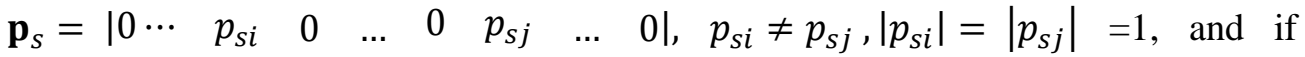
$p_{s i}>0$ then $p_{s j}<0$, if $p_{s i}<0$ then $p_{s j}>0$.

The matrix $\boldsymbol{\Sigma}$ is a symmetrical $N \times N$ square one with volatilities on its diagonal to the corresponding $i=1, \ldots, N$ assets

$$
\boldsymbol{\Sigma}=\left|\begin{array}{ccc}
\sigma_{11}^{2} & \ldots & c_{1 N} \\
\ldots & \ldots & \ldots \\
c_{N 1} & \ldots & \sigma_{N N}^{2}
\end{array}\right| .
$$

Using (36), the matrix $\boldsymbol{\Omega}$ will be diagonal one with components $\omega_{\mathrm{ss}}, s=1, \ldots, k$, Or

$$
\omega_{\mathrm{ss}}=\tau\left(\sigma_{s i}^{2}+\sigma_{s j}^{2}-2 c_{i j}\right), s=1, \ldots, k, i, j=1, \ldots, N .
$$

Relation (37) is compared with a portfolio, containing two assets with equal weights and negative correlation. Following [23], the volatility of this portfolio gives

$$
\sigma_{p}^{2}=0.5\left(\sigma_{1}^{2}+\sigma_{2}^{2}-2 c_{12}\right),
$$

where $\sigma_{1}^{2}, \sigma_{2}^{2}, c_{12}$ are the volatilities of assets 1 and 2 and the covariance between the returns of these two assets.

The comparison between relations (37) and (38) shows that the variation of the subjective view $\omega_{\mathrm{ss}}$ is proportional to the risk of a virtual portfolio, which contains only two assets with equal weights. But assuming different weights for the subjective views the vector $\mathbf{p}_{s}$ will have non integer components like

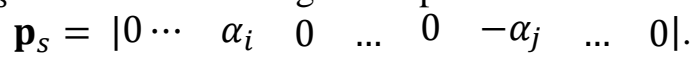

The values $\alpha_{i}$ and $\alpha_{j}$ must satisfy the normalization equation

$$
\left|\alpha_{i}\right|+\left|\alpha_{j}\right|=1
$$

The modification (39) makes the matrix $\boldsymbol{\Omega}$ with new components

$$
\omega_{\mathrm{ss}}=\tau\left(\alpha_{\mathrm{i}}^{2} \sigma_{s i}^{2}+\alpha_{\mathrm{j}}^{2} \sigma_{s j}^{2}-2 \alpha_{i} \alpha_{j} c_{i j}\right) \text {. }
$$

In [24] $\alpha_{i}$ is assumed to be the maximal difference between the historical average return $\mathrm{E}_{i}$ and the implied return $\Pi_{i}$, normalized by the asset volatility

$$
\text { if } \alpha_{i}>0 \text { then } \max _{i}\left(\frac{\Pi_{i}-\mathrm{E}_{i}}{\sigma_{i}^{2}}\right), i=1, \ldots, N \text {. }
$$

For the case (42) the asset $i$ is underestimated and it is expected its mean return to increase. Respectively, $j$ is defined from the minimal value if the relation holds

$$
\text { if } \alpha_{j}<0 \text { then } \min _{j}\left(\frac{\Pi_{j}-\mathrm{E}_{j}}{\sigma_{j}^{2}}\right) \text {. }
$$

For this case $j$ is overestimated and it is expected its mean return to decrease. The s-th component of matrix $\mathbf{Q}, q_{s}$ is assumed to have value according to (44),

$$
q_{s}=\max _{i}\left(\Pi_{i}-\mathrm{E}_{i}\right), \quad i=1,2, \ldots, N .
$$


The notation used in the this case of weighted views is $\mathbf{P}(\alpha)$, because matrix $\mathbf{P}$ contains algebraic values of $\alpha_{i}$, which satisfy the normalization Equation (40).

\section{Application of BL model for active portfolio management}

The modification of the expert views for the BL model are applied for active management. Small set of 5 technological companies are used: APPLE, GOOGLE, AMAZON, MICROSOFT and FACEBOOK. Market data from January till July 2018 have been used from [29]. The average monthly closing prices are the initial data for the definition of the portfolio problem. The returns of the assets for the first six months of 2018 are used as historical data. These data are used for the evaluation of the mean assets returns and the covariance matrix. They give the initial parameters for the classical mean variance portfolio model and for BL one: matrices $\mathbf{E}_{\mathrm{MV}}$ and $\boldsymbol{\Sigma}_{\mathrm{MV}}$. Additionally, from these historical data, the parameters of matrices $\mathbf{P}(\alpha)$ and $\boldsymbol{\Omega}$ are evaluated. Thus, both problems MV and BL are well numerically defined. Their solutions $\mathbf{w}_{\mathrm{MV}}^{\mathrm{opt}}$ (for MV problem) and $\mathbf{w}_{\mathrm{BL}}^{\text {opt }}$ for (BL model) are applied for the next, future month of July 2018. In the end of month of July, having the real data about the assets returns $\mathbf{E}_{\mathbf{r}}$ and covariation matrix $\sum_{\mathbf{r}}$, the results of the investments with the weights $\mathbf{w}_{\mathrm{MV}}^{\mathrm{opt}}$ and $\mathbf{w}_{\mathrm{BL}}^{\mathrm{opt}}$ are compared. The portfolio returns and risks with these two sets of weights are evaluated, Fig 2.

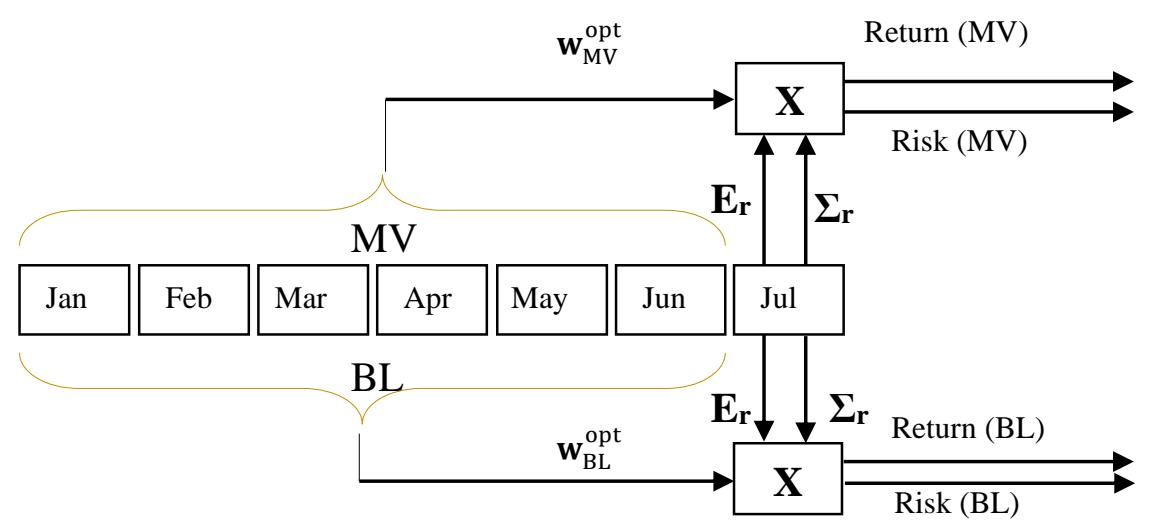

Fig. 2. Common base for the evaluation of MV and BL portfolio models

Thus, starting with the same set of data for the historical period, applying the classical MV portfolio and the modified BL models, it is possible to assess the benefit from these two investment decisions. For implementation of active portfolio management, the next investment period will use as historical data these one from February to July (rolling 6 months period). The new evaluated weights will be applied for the next, future month of August. Hence, the active strategy by using 6 months historical data, after evaluation of $\mathbf{w}_{\mathrm{MV}}^{\mathrm{opt}}$ and $\mathbf{w}_{\mathrm{BL}}^{\mathrm{opt}}$, they are applied for the next future month. To be able the reader to check and to follow the computations, the algorithm in the next section is illustrated by calculations only for the first investment period: 
the historical period is from January to June and the investment decisions and comparisons are done for the month of July.

\section{Algorithm for active portfolio management with modified BL model}

The initial data of the technological companies January-July 2018 are the following: APL $=[-3.658677351 ; \quad 4.090484081 ; \quad-2.671637211 ; 9.245795939$; $1.662532915 ; 0.895974703 ; \mathbf{1 2 . 1 0 3 6 6 5 8 6}]$;

GOOG $=[-4.170135971 ; \quad 0.173009734 ; \quad-4.920339419 ; \quad 3.356001965 ;$ $6.928137123 ; 4.519526778 ; \mathbf{3 . 2 7 9 0 5 5 1 9 4}]$;

AMAZ $=[10.18724871 ; \quad 6.794716245 ; \quad-4.68376233 ; \quad 8.628347616$; 6.219594006; 5.344953989; 6.343110781];

MICR=[1.43508572; 1.675810417; 0.339846685; 4.040778655; 3.691988306; 4.053889582; 3.866427325];

FACE $=[-2.537473141 ; \quad-3.761009093 ; \quad-5.579037757 ; \quad 11.67173454 ;$ $6.744448251 ; 2.406708566 ;-\mathbf{1 1 . 2 3 9 3 3 1 6 8}]$.

The boldface values are the future mean returns of the assets for the month of July. The risk free value has been taken according to the American interest rate (Fed) from [30], $r_{f}=2.5 \%$. The two portfolio problems, based on MV and BL models use 6 month historical data and evaluate the weights of their optimal solutions $\mathbf{w}_{\mathrm{MV}}^{\mathrm{opt}}$ and $\mathbf{w}_{\mathrm{BL}}^{\text {opt }}$. These weights are applied for the evaluation of the portfolios risk and return but with the real returns $\mathbf{E}_{\mathbf{r}}$ and $\sum_{\mathbf{r}}$ for month of July 2018 (the boldface noted values from the initial data given above). The portfolio return and risk for MV model is noted as Return(MV) and, Risk(MV), respectively for the BL model as Return(BL) and Risk(BL)

$$
\begin{array}{ll}
\operatorname{Return}(\mathrm{MV})=\mathbf{E}_{\mathbf{r}}^{\mathrm{T}} \mathbf{w}_{\mathrm{MV}}^{\mathrm{opt}}, & \operatorname{Risk}(\mathrm{MV})=\mathbf{w}_{\mathrm{MV}}^{\mathrm{optT}} \sum_{\mathbf{r}} \mathbf{w}_{\mathrm{MV}}^{\mathrm{opt}}, \\
\operatorname{Return}(\mathrm{BL})=\mathbf{E}_{\mathbf{r}}^{\mathrm{T}} \mathbf{w}_{\mathrm{BL}}^{\mathrm{opt}}, & \operatorname{Risk}(\mathrm{BL})=\mathbf{w}_{\mathrm{BL}}^{\mathrm{optT}} \sum_{\mathbf{r}} \mathbf{w}_{\mathrm{BL}}^{\mathrm{opt}},
\end{array}
$$

The matrix $\sum_{\mathbf{r}}$ is the actual covariance matrix, evaluated using data for the 6-month period from February till July 2018. The evaluations are performed first for the MV portfolio problem and then for the BL one. The MATLAB software suit is used.

5.1. Definition and solution of MV portfolio problem

1. Evaluation of the average historical returns $\mathbf{E}_{\mathrm{MV}}$. For the period of the first 6 months for the assets returns gives,

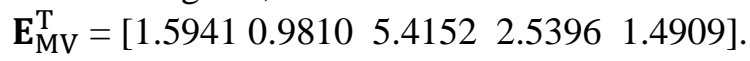

2. Evaluation of the historical covariance matrix. For the historical 6 months it is

$$
\begin{aligned}
& \Sigma_{\mathrm{MV}}=\left[\begin{array}{lllll}
22.2121 & 13.2647 & 9.2294 & 4.7074 & 23.1657 ;
\end{array}\right. \\
& \begin{array}{llllll}
13.2647 & 23.1086 & 9.2136 & 7.0292 & 25.0760 ;
\end{array} \\
& \begin{array}{lllll}
9.2294 & 9.2136 & 27.5282 & 4.2794 & 16.3609 ;
\end{array} \\
& \begin{array}{lllll}
4.7074 & 7.0292 & 4.2794 & 2.5359 & 9.4524 ;
\end{array} \\
& 23.1657 \quad 25.0760 \quad 16.3609 \quad 9.4524 \quad 45.1764] \text {. }
\end{aligned}
$$

These initial data define the MV portfolio problem, which is solved 


$$
\begin{gathered}
\min _{\mathbf{w}}\left[\delta \mathbf{E}_{\mathrm{MV}}^{\mathrm{T}} \mathbf{w}-(1-\delta) \mathbf{w}^{\mathrm{T}} \sum_{\mathrm{MV}} \mathbf{w}\right], \mathbf{w}^{\mathrm{T}} \mathbf{1}=\mathbf{1}, \mathbf{w}^{\mathrm{T}}=\left(w_{1}, \ldots, w_{N}\right) \geq 0, \\
\mathbf{1}^{\mathrm{T}}=\left|\begin{array}{lll}
1 & \cdots & 1
\end{array}\right|_{N \times 1} .
\end{gathered}
$$

3. Evaluation of the "efficient frontier". By changing $\delta, 0 \leq \delta \leq 1$, $\delta=0: 0.01: 1$, the "efficient frontier" is defined by $101 \mathrm{MV}$ problem solutions and its graphical presentation is given in Fig. 3. The portfolio characteristics $\operatorname{Returun}_{\mathrm{MV}}(i)$ and $\operatorname{Risk}_{\mathrm{MV}}(i), i=1, \ldots, 101$ are found. The values of the Excess_Sharp_ratio ${ }_{\mathrm{MV}}(i)$, $i=1, \ldots, 101$, are evaluated by the relation

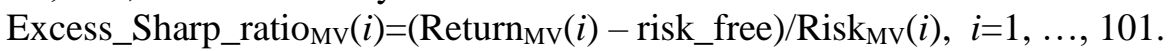

The portfolio solutions of the weights $\mathbf{w}_{\mathrm{MV}}^{\text {opt }}$ are kept in a matrix with dimension $101 \times 5$. The relation Exess_Sharp_ratio ${ }_{M v}$ from Risk ${ }_{M v}$ is given in Fig. 4.

4. Evaluation of the maximal value of the Excess Sharp ratio. This gives

$$
\max _{i=1, \ldots, 101}\left[\text { Excess_Sharp_ratio }_{\mathrm{MV}}(i)\right]=0.1640, i_{\max }=87 .
$$

and the corresponding weights of this portfolio are

$$
\mathbf{w}_{\mathrm{MV}}^{\mathrm{optT}}\left(i_{\max }\right)=\left[\begin{array}{lllll}
0 & 0.0000 & 0.3296 & 0.6704 & 0
\end{array}\right] .
$$

These portfolio weights will be invested for the next, future month of July.

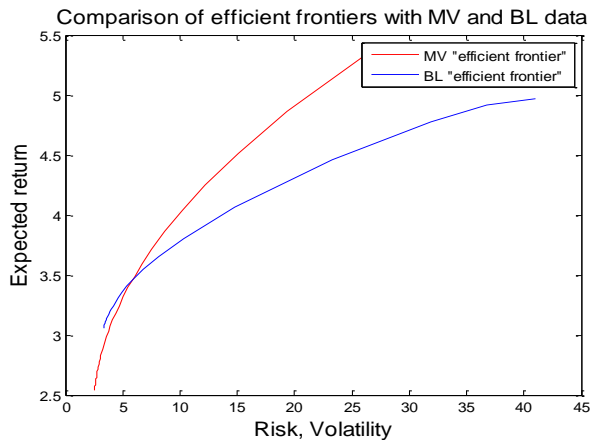

Fig. 3. Comparison of the efficient frontiers for the MV and BL portfolios for MV and BL cases

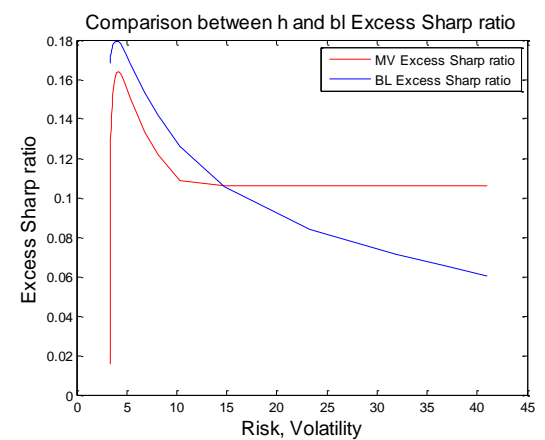

Fig. 4. Comparison of the portfolio characteristics of Excess Sharpe ratio

\subsection{Definition and solution of the BL portfolio problem}

The BL model first evaluates the "implied" assets returns. For that case the characteristics of this particular market with 5 assets must be evaluated. This specific market does not allow world financial indices to be used. The specific market is given by the tangent point between the Capital Market Line and the efficient frontier. The tangent line must pass through the risk free point. Analytically, the new market point in this research is found from system of two equations: the quadratic analytical approximation of the efficient frontier and a tangent line toward the efficient frontier, which passes through the risk-free point.

1. Analytical approximation of the $M V$ "efficient frontier", numerically evaluated by 101 points from $\mathrm{p} .3$ of the MV portfolio case. The approximation of the MV "efficient frontier" is done by a quadratic curve

$$
y=a_{2} x^{2}+a_{1} x+a_{0}
$$


where $y$ is the portfolio Return and $x$ is the portfolio Risk. Least square method for this approximation is used to evaluate the unknown coefficients $a_{0}, a_{1}, a_{2}$ from the linear equation system

$$
\left|\begin{array}{ccc}
n & \sum_{i=1}^{n} x_{i} & \sum_{i=1}^{n} x_{i}^{2} \\
\sum_{i=1}^{n} x_{i} & \sum_{i=1}^{n} x_{i}^{2} & \sum_{i=1}^{n} x_{i}^{3} \\
\sum_{i=1}^{n} x_{i}^{2} & \sum_{i=1}^{n} x_{i}^{3} & \sum_{i=1}^{n} x_{i}^{4}
\end{array}\right|\left|\begin{array}{c}
a_{o} \\
a_{1} \\
a_{2}
\end{array}\right|=\left|\begin{array}{c}
\sum_{i=1}^{n} y_{i} \\
\sum_{i=1}^{n} y_{i} x_{i} \\
\sum_{i=1}^{n} y_{i} x_{i}^{2}
\end{array}\right|,
$$

where the values of the portfolio risk are $x_{i}$, and for the portfolio return are $y_{i}$, $\left(x_{i}, y_{i}\right), n=101$ points from the efficient frontier.

Graphically, the MV portfolios of the "efficient frontier" and its quadratic approximation are given in Fig. 5. The values of the coefficients are calculated to $\left[\begin{array}{lll}a_{2} & a_{1} & a_{0}\end{array}\right]^{\mathrm{T}}=\left[\begin{array}{lll}-0.0045 & 0.2394 & 2.0917\end{array}\right]^{\mathrm{T}}$. Because the tangent point with the Capital Market Line is needed for the evaluation of the market characteristics, one needs good approximation only for the area, where the market point is expected. This consideration can help for decrease the number $n$ of the efficient frontier.

2. Analytical evaluation of the market point $\left(y_{M}, x_{M}\right)$. The market point is a tangent one between the curve of the approximated MV "efficient frontier" and the Capital Market Line. The latter also has to pass through the point of the risk free asset, $\left(0, r_{f}=2.5 \%\right)$. Using relations from the analytical geometry, the tangent line $t$ towards the MV "efficient frontier" must have analytical description

$$
t \equiv y-y_{M}=\frac{d y}{d x}\left(y_{M}, x_{M}\right)\left(x-x_{M}\right)
$$

where

$$
\frac{d y}{d x}\left(y_{M}, x_{M}\right)=2 a_{2} x_{M}+a_{1},
$$

and the tangent point $\left(y_{M}, x_{M}\right)$ give the parameters of the unknown market point.

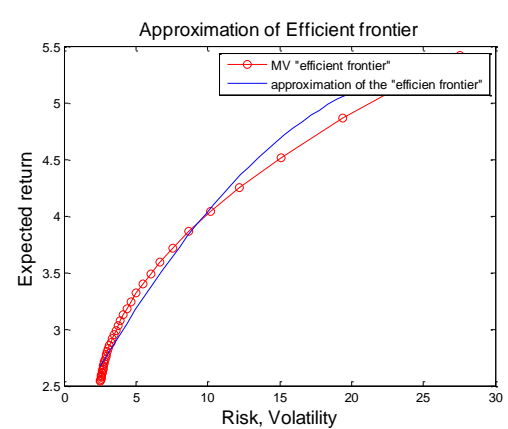

Fig. 5. Graphical presentation of the MV "efficient frontier" and its quadratic approximation

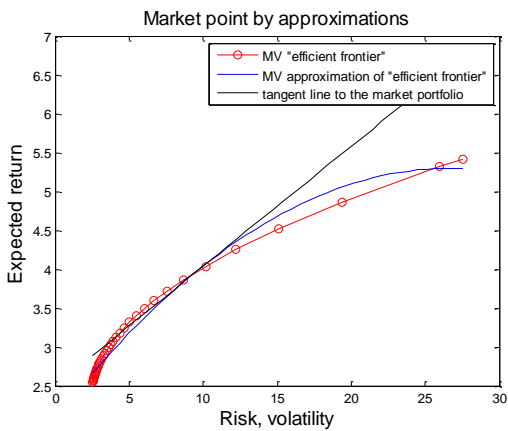

Fig. 6. Graphical presentation of the tangent line to the market portfolio

Because the tangent line $t$ passes through the risk free point, hence it holds

$$
t\left(0, r_{f}\right) \equiv r_{f}-y_{M}=2 a_{2} x_{M}+a_{1}\left(0-x_{M}\right) .
$$

Both equations of the tangent line (47) and the approximation of the MV "efficient frontier" (46) define two equations with two unknown, $y_{M}=E_{M}$ and $x_{M}=\sigma_{M}^{2}$ :

$$
\begin{gathered}
t\left(0, r_{f}\right) \equiv r_{f}-y_{M}=2 a_{2} x_{M}+a_{1}\left(0-x_{M}\right) \\
y(x) \equiv y_{M}=a_{2} x_{M}{ }^{2}+a_{1} x_{M}+a_{0} .
\end{gathered}
$$



found

From these two equations explicit analytical relation for the market risk $\sigma_{M}^{2}$ is

$$
x_{M}=\sqrt{\frac{a_{0}-r_{f}}{a_{2}}}=\sigma_{M}^{2} .
$$

The market return $y_{M}=E_{M}$ is calculated from (46) for given $x_{M}$. Using these values for the market point this gives $\left(y_{M}=E_{M}=3.973, x_{M}=\sigma_{M}^{2}=9.5626\right)$. The graphical presentation of the tangent line is given in Fig. 6.

3. Evaluation of the market weights. These weights will show market

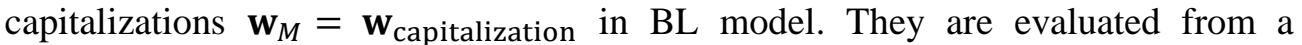
classical Markowitz problem for minimization of the portfolio risk, but keeping the portfolio return on the level of $y_{M}=\sigma_{M}^{2}=3.973$. The formal constrained optimization problem is

$$
\min _{\mathbf{w}}\left[\mathbf{w}^{\mathrm{T}} \sum_{\mathrm{MV}} \mathbf{w}\right], \mathbf{E}_{\mathrm{MV}}^{\mathrm{T}} \mathbf{w}=y_{M}, \mathbf{w}^{\mathrm{T}} \mathbf{1}=\mathbf{1}, \mathbf{w}^{\mathrm{T}}=\left(w_{1}, \ldots, w_{N}\right) \geq 0, \mathbf{1}=\left|\begin{array}{c}
1 \\
1_{N \times 1}
\end{array}\right|_{N} .
$$

The solution of this problem gives

$$
\mathbf{w}_{M}^{\mathrm{T}}=\mathbf{w}_{\text {capitalization }}^{\mathrm{T}}=\left[\begin{array}{lllll}
0 & 0 & 0.4985 & 0.5015 & 0
\end{array}\right]^{\mathrm{T}} .
$$

4. Evaluation of the risk aversion parameter. From (18), this gives $\lambda=0.1540$.

5. Evaluation of the excess implied returns $\boldsymbol{\Pi}^{*}$, and the implied returns $\boldsymbol{\Pi}$ from (19). This gives

$$
\begin{gathered}
\boldsymbol{\Pi}^{* \mathrm{~T}}=\left[\begin{array}{lllll}
1.0723 & 1.2505 & 2.4443 & 0.5245 & 1.9865
\end{array}\right]^{\mathrm{T}} \text { and } \\
\boldsymbol{\Pi}^{\mathrm{T}}=\left[\begin{array}{lllll}
3.5723 & 3.7505 & 4.9443 & 3.0245 & 4.4865
\end{array}\right]^{\mathrm{T}} .
\end{gathered}
$$

6. Definition of vectors $\mathbf{P}$ and $\mathbf{Q}$. The subjective views are defined from (42), (43) and (44). Using assets returns $\mathrm{E}_{\mathrm{MV}}(i)$ and assets $\mathrm{E}_{\mathrm{MV}}(i)$ risks $\sigma_{i}^{2}, i=1, \ldots, N$, the components of the weighted vector $\mathbf{P}(\boldsymbol{\alpha})$ are

$$
\begin{gathered}
\alpha_{i}=0.8652=\max _{i}\left(\frac{\Pi_{i}-E_{i}}{\sigma_{i}^{2}}\right), i=2 ; \quad \alpha_{j}=-0.1348=\min _{j}\left(\frac{\Pi_{j}-E_{j}}{\sigma_{j}^{2}}\right), j=3, \\
q_{2}=\left(\Pi_{2}-\mathrm{E}_{2}\right)=0.4709,
\end{gathered}
$$

\begin{tabular}{|c|c|c|c|c|}
\hline $\mathbf{E}_{\mathrm{BL}}^{\mathrm{T}}=[3.6347$ & 3.8648 & 4.9703 & 3.0581 & 4.6 \\
\hline $\mathrm{s}=[31.6455$ & 16.8318 & 13.1475 & 6.1613 & 31.5626 \\
\hline 6.8318 & 29.0457 & 12.5438 & 8.8948 & 755 \\
\hline 16.8 & 29.0 & 12.5 & 8.8948 & 55 \\
\hline 13.1 & 12.5 & 41.0022 & 43 & 23. \\
\hline 6.161 & 8.8948 & 6.0443 & 3.3198 & 12.4 \\
\hline 1.562 & 31.7755 & 23.2145 & 12.4647 & 61.6 \\
\hline
\end{tabular}

which defines the vector $\mathbf{P}=\left[\begin{array}{lllll}0 & 0.8652 & -0.1348 & 0 & 0\end{array}\right]$. Because only one view is made, $\mathbf{P}$ is a vector and $\mathbf{Q}=q_{3}$ is a unique value.

7. Evaluations of the BL mean returns and covariance matrix from (27). The parameter $\tau$ must be less than 1 and here it is chosen $\tau=0.5$. From (26), it follows:

8. Evaluation of the BL "efficient frontier" with the data of p. 7. A set of optimization problems is solved as in Step 3 of Paragraph 5.1. New "efficient frontier" is evaluated. Both frontiers for MV and BL cases are given in Fig. 3. The corresponding values of the Excess_Sharp_ratio ${ }_{\mathrm{BL}}(i), i=1, \ldots, 101$, are evaluated by 
the same way as in Step 3. The portfolio solutions of the weights $\mathbf{w}_{\mathrm{BL}}$ are kept in a matrix with dimension $101 \times 5$.

9. Evaluation of the maximum value of the Excess Sharp ratio. For BL case $\max _{i=1, \ldots, 101}\left[\right.$ Excess_Sharp_ratio $\left._{\mathrm{BL}}(i)\right]=0.1795, i_{\mathrm{max}}=86$,

and weights are: $\quad \mathbf{w}_{\mathrm{BL}}^{\mathrm{opt}^{\mathrm{T}}}\left(i_{\max }\right)=\left[\begin{array}{lllll}0 & 0.0000 & 0.0836 & 0.9164 & 0\end{array}\right]$.

These weights $\mathbf{w}_{\mathrm{BL}}^{\mathrm{opt}^{\mathrm{T}}}=\mathbf{w}_{\mathrm{BL}}^{\mathrm{opt}^{\mathrm{T}}}\left(i_{\max }\right)$ will be applied for the month of July.

\section{Comparison of the MV and the modified BL portfolio models.}

The portfolios weights $\mathbf{w}_{\mathrm{MV}}^{\mathrm{opt}}$ and $\mathbf{w}_{\mathrm{BL}}^{\mathrm{opt}}$ are chosen from the corresponding "efficient frontiers" of MV and BL models. These portfolios correspond to the "best" case, where the Excess Sharp ratio has a maximum. The comparison is based on the application of these weights for the future month July. In the end of July the portfolio returns and risks are assessed with the calculated prior $\mathbf{w}_{\mathrm{MV}}^{\mathrm{opt}}$ and $\mathbf{w}_{\mathrm{BL}}^{\mathrm{opt}}$ and the posterior evaluated real asset returns $\mathbf{E}_{\mathbf{r}}$ and risk $\boldsymbol{\Sigma}_{\mathbf{r}}$. The corresponding portfolio characteristics with the modified BL and MV models are assessed and compared.

1. Estimation of the real mean return and covariation matrix for the month of July. These real posterior data of asset returns, noted in bold for July are given as $\mathbf{E}_{\mathbf{r}}^{\mathrm{T}}=\left[\begin{array}{llllll}12.10366586 & 3.279055194 & 6.343110781 & 3.866427325 & -11.23933168\end{array}\right]$.

The new covariance matrix for the actual six months from February to July is

$$
\begin{aligned}
& \Sigma_{\mathrm{r}}=\left[\begin{array}{llllll}
34.2889 & 14.5041 & 9.0843 & 5.9150 & 0.1920
\end{array}\right. \\
& \begin{array}{llllll}
14.5041 & 20.0116 & 7.9826 & 6.2933 & 16.7175
\end{array} \\
& \begin{array}{llllll}
9.0843 & 7.9826 & 23.0631 & 3.7420 & 11.9465
\end{array} \\
& \begin{array}{lllll}
5.9150 & 6.2933 & 3.7420 & 2.3648 & 5.4640
\end{array} \\
& \left.\begin{array}{lllll}
0.1920 & 16.7175 & 11.9465 & 5.4640 & 60.7983
\end{array}\right] \text {. }
\end{aligned}
$$
July.

The evaluated weights $\mathbf{w}_{\mathrm{MV}}^{\mathrm{opt}}$ and $\mathbf{w}_{\mathrm{BL}}^{\mathrm{opt}}$ define two investment decisions for

2. Evaluation of portfolios Risks and Returns with the new mean return data $\mathbf{E}_{\mathbf{r}}^{\mathbf{T}}$ and covariance matrix $\boldsymbol{\Sigma}_{\mathbf{r}}$. Relations (45) are applied and the portfolio characteristics for the MV and modified BL models one are kept. The rolling procedure for investment is applied till month of November 2018. The average results for investment from July until November are given on Table.1 It is seen that the returns of MV and BL portfolios are very close. The MV model provides a bit higher return.

\begin{tabular}{|c|c|c|c|}
\hline \multirow[t]{2}{*}{ MV optimization } & $\begin{array}{c}\text { Return }(\mathrm{MV})= \\
\mathbf{E}_{\mathbf{r}}^{\mathrm{T}} \mathbf{w}_{\mathrm{MV}}^{\mathrm{opt}}\end{array}$ & $\begin{array}{c}\operatorname{Risk(MV)}= \\
\mathbf{w}_{\mathrm{MV}}^{\text {opt T }} \boldsymbol{\Sigma}_{\mathbf{r}} \mathbf{w}_{\mathrm{MV}}^{\text {opt }}\end{array}$ & $\mathbf{w}_{\mathrm{MV}}^{\mathrm{opt}}$ \\
\hline & 4.6828 & 5.2225 & {$\left[\begin{array}{llll}0 & 0 & 0.3296 & 0.6704\end{array}\right]$} \\
\hline \multirow[t]{2}{*}{ BL optimization } & $\begin{array}{c}\text { Return }(\mathrm{BL})= \\
\mathbf{E}_{\mathbf{r}}^{\mathrm{T}} \mathbf{w}_{\mathrm{BL}}^{\text {opt }}\end{array}$ & $\begin{array}{c}\operatorname{Risk(BL)=}= \\
\mathbf{w}_{\mathrm{BL}}^{\text {opt T }} \boldsymbol{\Sigma}_{\mathbf{r}} \mathbf{w}_{\mathrm{BL}}^{\text {opt }}\end{array}$ & $\mathbf{w}_{\mathrm{BL}}^{\mathrm{opt}}$ \\
\hline & 4.0734 & 2.7202 & {$\left[\begin{array}{llll}0 & 0 & 0.0836 & 0.9164\end{array}\right]$} \\
\hline
\end{tabular}
However, the risk of MV portfolio is considerably higher according to the BL one. Hence having close values of returns but very different level of risk, the BL model gives preferences for the active management of portfolio investments.

Table 1. Comparison of the MV and BL portfolios 
This procedure for evaluations was sequentially applied in time, which gives an active portfolio policy. Advantages of this algorithm comes from the opportunity to design a portfolio in a particular market with a small number of assets, to estimate the market characteristics of this particular market, and to use additional information from the historical data for the definition of the expert views. Thus, comparison between BL and MV portfolio models can be done, on the basis of common initial data for the investment.

\section{Conclusions}

The paper makes an analytical overview of the formal relations, which are needed for the definition of the BL portfolio model. The last is based on the usage of the Markowitz portfolio models and the formal relations originated from the Capital Asset Pricing Theory. The BL portfolio model needs as input data the parameters of the market point and the set of parameters from expert views. The market point, given by world market indices cannot be used in case of limited set of assets in the portfolio, because new particular small market is formed. This lack of market characteristics in this research is solved by definition of a set of equations with quadratic approximation for the efficient frontier and additional linear relation for the tangent line towards the efficient frontier, which passes through the risk-free point. Additionally, new formalization of the expert views is applied, which is based on assessment of the difference between the implied and historical mean returns of the assets. Thus, the original BL model has been modified by means to overcome the requirement for having subjective views. The BL modification in the form of expert views allows the two general portfolio models, MV and $\mathrm{BL}$ one to be compared because they use common initial information for the definition of the portfolio problems. The modification of the BL model has been performed for the parameters of the expert views by introducing weighted form of matrix $\mathbf{P}$. The application of an active policy of portfolio management based on BL model has been illustrated, applying rolling horizon for portfolio management. This policy of sequential application of the modified BL model for active portfolio management gives better benefits in comparison with the classical MV model. The experimental results illustrate better performance of the applied modified BL model.

Acknowledgments: This work has been supported by project ДН12/10, 20.12.2017 of the Bulgarian National Science Fund "Integrated bi-level optimization in information service for portfolio optimization".

\section{References}

1. A 11 a j, E. The Black-Litterman Model and Views from a Reverse Optimization Procedure: An Outof-Sample Performance Evaluation. - Article in SSRN Electronic Journal, 2017. DOI: $10.2139 / \mathrm{ssrn} .2999335$. 
2. A 11 e n, D., C. Li z i e ri, S. S a t c he 11. In Defense of Portfolio Optimization: What If We Can Forecast? - Financial Analysts Journal, Vol. 75, 2019, No3, 20-38.

3. B artkowiak, M., A. Rutkowska. Black-Litterman Model with Multiple Experts Linguistic Views. - In: Soft Methods for Data Science. Springer, 2017.

4. B e r t s i m a s, D., V. G u p t a, I. C. P a s c h a 1 i d i s. Inverse Optimization: A New Perspective on the Black-Litterman Model. - J. Operation Research, Vol. 60, 11 December 2012, No 6, pp. 1389-1403.

5. B la ck, F., R. Litterman. Asset Allocation: Combining Investor Views with Market Equilibrium. - The Journal of Fixed Income, Vol. 1, 1991, No 2, p. 7-18.

6. W ing, Che ung. The Black-Litterman Model Explained. - Journal of Asset Management, Vol. 11, 2011, No 4, pp. 229-243.

7. Cui, X., J. Ga o, Y. Shi. Multi-Period Mean-Variance Portfolio Optimization with Management Fees. - Int J. Oper Res, 2019, pp. 1-22.

8. D a v i s, M., S. L 1 e o. A Simple Procedure to Incorporate Predictive Models in a Continuous Time Asset Allocation. - Quantitative Finance Letters, Vol. 4, 2016, No 1, pp. 40-46.

9. D u qi, L. F., G. T or lu c c i o. The Black-Litterman Model: the Definition of Views Based on Volatility Forecasts. - J. Applied Financial Economics, Vol. 24, 2014, No 19, pp. 12851296.

10. F a n g, Y., L. B o, D. Z h a o, S. W a n g. Fuzzy Views on Black-Litterman Portfolio Selection Model. - J. of Systems Science and Complexity, Vol. 31, 2018, No 4, pp. 975-987.

11. Ga o, Xuedong, Gu Kan. Determination of the Starting Point in Time Series for Trend Detection Based on Overlapping Trend. - Cybernetics and Information Technologies, Vol. 16, 2016, No 6, pp. 98-110.

12. Ge orgi eva, P. V. Genetic Fuzzy System for Financial Management. - Cybernetics and Information Technologies, Vol. 18, 2018, No 2, pp. 20-35.

13. Ge orgieva, P., I. P. Popchev, S. N. Stoyanov. - Cybernetics and Information Technologies, Vol. 15, No 3, 2015, pp. 41-51.

14. Ge yer, A., K. Lucivjanská. The Black-Litterman Approach and Views from Predictive Regressions: Theory and Implementation. - The Journal of Portfolio Management, Vol. 42, 2016, No 4, pp. 38-48

15. Harris, R. D. F., E. Stoja, L. Tan. The Dynamic Black-Litterman Approach to Asset Allocation. - European Journal of Operational Research, Vol. 259, 2017, pp. 1085-1096.

16. H e, G., R. L i t t e r m a n. The Intuition behind Black-Litterman Model Portfolios. - In: Investment Management Research. Goldman Sachs \& Company, 1999.

17. I d z o r e k, T. M. A Step-by-Step Guide to the Black-Litterman Model. 2002. https://faculty.fuqua.duke.edu/ charvey/Teaching/BA453_2006/Idzorek_onBL.pdf

18. Kolm, P. N., R. Tutuncu, F. J. Fabozzi. 60 Years of Portfolio Optimization: Practical Challenges and Current Trends. - European Journal of Operational Research, Vol. 234, 2014, pp. 356-371

19. K or s e m o v, D., D. B or i s s o v a, I. M u s t a k e r o v. Combinatorial Optimization Model for Group Decision-Making. - Cybernetics and Information Technologies, Vol. 18, 2018, No 2, pp. 65-73.

20. M a h m u t, K., U. A y d i n, K. A. B a r i s. A Hybrid Approach for Generating Investor Views in Black-Litterman Model. - Expert Systems with Applications, Vol. 128, 2019

21. M a r k o w it z, H. Portfolio Selection. - Journal of Finance, Vol. 7, 1952, pp.77-91.

22. M e u c c i, A. The Black-Litterman Approach: Original Model and Extensions. Bloomberg. - In: Alpha Research\&Education Paper, No 1, 2008.

23. M i c h a u d, R. O., D. N. E s c h, R. O. M i c h a u d. Deconstructing Black-Litterman: How to Get the Portfolio You Already Knew You Wanted. - SSRN Electronic J., Vol. 11, 2013, No 1, pp. 6-20.

24. S a t ch e 11, S., A. S c o w c r of t. A Demystification of the Black-Litterman Model: Managing Quantitative and Traditional Portfolio Construction. - J. of Asset Management, Vol. 1, 2000, pp. 138-150. 
25. S h a r p e, W. Portfolio Theory and Capital Market. McGraw Hill, 2000. 316 p.

26. V la di mi rov, M., T. S to ilov, K. S to ilova. New Formal Description of Expert Views of Black-Litterman Asset Allocation Model. - Cybernetics and Information Technologies, Vol. 17, 2017, No 4, pp. 87-98.

27. W a 1 t e r s, J. Reconstructing the Black-Litterman Model, 2014.

28. https://www.moneyworks4me.com/about-stock-market/portfolio-management

29. https://finance.yahoo.com

30. www.global-rated.com

Received: 07.04.2020; Second Version: 26.04.2020; Accepted: 21.05.2020 